\title{
Simple Unequal Allocation Procedure for Ranked Set Sampling with Skew Distributions
}

Dinesh Bhoj

Rutgers University, dbhoj@camden.rutgers.edu

Girish Chandra

Indian Council of Forestry Research and Education, gchandra23@yahoo.com

Follow this and additional works at: https://digitalcommons.wayne.edu/jmasm

Part of the Applied Statistics Commons, Social and Behavioral Sciences Commons, and the Statistical Theory Commons

\section{Recommended Citation}

Bhoj, D., \& Chandra, G. (2019). Simple unequal allocation procedure for ranked set sampling with skew distributions. Journal of Modern Applied Statistical Methods, 18(2), eP2811. doi: 10.22237/jmasm/ 1604189700

This Regular Article is brought to you for free and open access by the Open Access Journals at DigitalCommons@WayneState. It has been accepted for inclusion in Journal of Modern Applied Statistical Methods by an authorized editor of DigitalCommons@WayneState. 


\section{Simple Unequal Allocation Procedure for Ranked Set Sampling with Skew Distributions}

\section{Cover Page Footnote}

Acknowledgment: The authors thank the unknown Reviewers and the Editor for their useful comments and suggestions on the earlier version of this paper 


\title{
Simple Unequal Allocation Procedure for Ranked Set Sampling with Skew Distributions
}

\author{
Dinesh Bhoj \\ Rutgers University \\ Camden, NJ
}

\author{
Girish Chandra \\ Indian Council of Forestry Research and Education \\ Dehradun, India
}

\begin{abstract}
A practical unbalanced Ranked Set Sampling (RSS) model is proposed to estimate the population mean of positively skewed distributions. The gains in the relative precisions of the population mean based on the proposed model for chosen distributions are uniformly higher than those based on balanced RSS and the $t$-model proposed in Kaur et al. (1997). The relative precisions of the simple unequal allocation model are, with one exception, better than $(s, t)$-model which is better than $t$-model. The relative precision of the proposed model is very close or equal to the optimal Neyman allocation model.
\end{abstract}

Keywords: Gamma distribution, lognormal distribution, Pareto distribution, relative precision, skew distributions with heavy right tails, unequal allocation model, Weibull distribution

\section{Introduction}

Ranked Set Sampling (RSS), introduced by McIntyre (1952), is useful in estimating pasture and forage yields. It uses the observational economy by identifying a large number of sampling units from the population of interest and then quantify carefully selected subsample. It is a cost-efficient alternative to simple random sampling (SRS) if observations can be ranked according to the characteristic under investigation by means of visual inspection or other methods not requiring actual measurements. The RSS procedure has been used advantageously in agriculture, forestry, environmental, and ecological sampling where the exact measurement of unit is either difficult or expensive; see Halls and Dell (1966), Cobby et al. (1985), Stokes and Sager (1988), Johnson et al. (1993). For an overview of RSS, see Patil et al. (1994) and Chen et al. (2004).

doi: 10.22237/jmasm/1604189700 | Accepted: March 24, 2018; Published: September 4, 2020.

Correspondence: Girish Chandra, gchandra23@yahoo.com 


\section{BHOJ \& CHANDRA}

Dell and Clutter (1972) and Takahasi and Wakimoto (1968) provided mathematical foundations for RSS. Dell and Clutter also showed that the estimator for population mean based on RSS is at least as efficient as the estimator based on SRS with the same number of measurements even when there are ranking errors. Bhoj (2001) introduced RSS with unequal samples. Bhoj and Kushary (2016) proposed RSS with unequal samples for positively skewed distributions with heavy right tails. RSS is a non-parametric method. However, recently RSS has been used in the parametric setup; see Bhoj and Ahansullah (1996), Bhoj (1997), Lam et al. (1994), and Stokes (1995).

The selection of ranked set sample of size $k$ involves drawing $k$ random samples with $k$ observations in each sample. The $k$ units in each sample are ranked by using judgment or other inexpensive methods. The unit with the lowest rank is measured from the first sample, the unit with the second lowest rank is measured from the second sample, and this procedure is continued until the unit with the highest rank is measured from the last sample. The $k^{2}$ ordered observations in $k$ samples can be displayed as:

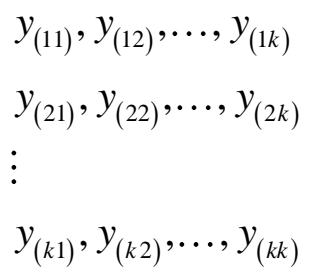

Measure only $k\left(y_{(i i)}, i=1,2, \ldots, k\right)$ observations and they constitute the RSS. These $k$ observations are independently but not identically distributed. In RSS, $k$ is usually small to reduce the ranking errors and therefore, to increase the sample size, the above procedure is repeated $m \geq 2$ times to get the sample of size $n=m k$. This RSS is called balanced RSS or RSS with equal allocation.

Under equal allocation, RSS is more precise method than SRS. However, the gain in the performance of the RSS can be improved when an appropriate unequal allocation is made rather than equal allocation. The resulting RSS procedure is called unbalanced RSS or RSS with unequal allocation. McIntyre (1952) proposed that the sample size corresponding to each rank order should be proportional to the standard deviation. This is also known as unequal allocation based on the approach of Neyman (1934). The implementation of Neyman's optimal allocation becomes elusive since in most cases standard deviations of order statistics are unavailable. Kaur et al. (1997) also proposed appropriate allocation models, called $t$-model and $(s, t)$-model. 


\section{UNEQUAL ALLOCATION FOR RSS FOR SKEW DISTRIBUTIONS}

The main interest of the current paper is to estimate the population mean of positively skew distributions with longer right tail. A simple unequal allocation model is proposed and compared with the approaches of Kaur et al. (1997) and Neyman (1934).

\section{Estimation of Mean}

Consider the balanced RSS to estimate the population mean. Let $Y_{(i: k) j}, i=1,2, \ldots, k$, $j=1,2, \ldots, m$ denote the value of the characteristic under study of $i^{\text {th }}$ out of $k$ order statistics in the $j^{\text {th }}$ cycle. The mean and variance of the $i^{\text {th }}$ rank order statistic are denoted by $\mu_{(i: k)}$ and $\sigma_{(i: k)}^{2}$, respectively. We denote the population mean and variance by $\mu$ and $\sigma^{2}$, respectively. Then the unbiased estimator for $\mu$ based on balanced RSS is given by

$$
\bar{Y}_{(k) \mathrm{eql}}=\frac{1}{k m} \sum_{i=1}^{k} \sum_{j=1}^{m} Y_{(i: k) j}
$$

with the variance

$$
\operatorname{Var}\left(\bar{Y}_{(k) \mathrm{eql}}\right)=\frac{1}{k^{2} m} \sum_{i=1}^{k} \sigma_{(i: k)}^{2}
$$

The relative precision of this estimator compared to the estimator based on SRS with same number of observations $n=k m$ is

$$
R P_{\mathrm{eq} 1}=\frac{\sigma^{2}}{\frac{1}{k} \sum_{i=1}^{k} \sigma_{(i: k)}^{2}}=\frac{\sigma^{2}}{\overline{\sigma^{2}}},
$$

where

$$
\frac{1}{k} \sum_{i=1}^{k} \sigma_{(i: k)}^{2}=\overline{\sigma^{2}}
$$

is the average within-rank variance. 


\section{BHOJ \& CHANDRA}

Consider unbalanced RSS where the allocations $m_{i}\left(m_{i} \geq 1\right)$ are used corresponding to the $i^{\text {th }}$ rank, $i=1,2, \ldots, k$. The sample size is

$$
n=\sum_{i=1}^{k} m_{i}
$$

Then the unbiased estimator for $\mu$ based on unbalanced RSS is

$$
\bar{Y}_{(k) \text { ueql }}=\frac{1}{k} \sum_{i=1}^{k} \frac{T_{i}}{m_{i}}
$$

where

$$
T_{i}=\sum_{j=1}^{m_{i}} Y_{(i: k) j}
$$

The variance of $\bar{Y}_{(k) \text { ueql }}$ is

$$
\operatorname{Var}\left(\bar{Y}_{(k) \text { ueql }}\right)=\frac{1}{k^{2}} \sum_{i=1}^{k} \frac{\sigma_{(i: k)}^{2}}{m_{i}}
$$

The optimal allocation model of Neyman (1934) is the same as the optimal allocation for RSS, and is given by

$$
m_{i}=\frac{n \sigma_{(i: k)}}{\sum_{i=1}^{k} \sigma_{(i: k)}}
$$

The minimum variance obtained by using (6) in (5) is

$$
\operatorname{Var}\left(\bar{Y}_{(k) \text { opt }}\right)=\frac{\bar{\sigma}^{2}}{n}
$$

where 
UNEQUAL ALLOCATION FOR RSS FOR SKEW DISTRIBUTIONS

$$
\bar{\sigma}=\frac{1}{k} \sum_{i=1}^{k} \sigma_{(i: k)}
$$

is the average within-rank standard deviation. The relative precision of Neyman's optimum allocation relative to SRS is given by

$$
R P_{\text {SRS:opt }}=\frac{\sigma^{2}}{\bar{\sigma}^{2}}
$$

and the relative precision of Neyman's optimum allocation relative to balanced RSS is

$$
R P_{\text {eqlopt }}=\frac{\overline{\sigma^{2}}}{\bar{\sigma}^{2}} .
$$

Due to the practical disadvantages in Neyman's optimal allocation model, Kaur et al. (1997) proposed two models namely, $t$-model and $(s, t)$-model. In the $t$-model, highest order statistic is measured $t$ times more frequently than the remaining order statistics. That is,

$$
m^{\prime} \equiv m_{1}=m_{2}=\ldots=m_{k-1}=\frac{m_{k}}{t}, \quad t \geq 1
$$

The sample size in this model is $n=(k-1+t) m^{\prime}$. The variance under this model is

$$
\operatorname{Var}\left(\bar{Y}_{(k) t}\right)=\frac{1}{k^{2} m^{\prime}}\left(a^{\prime}+b^{\prime} / t\right)
$$

where

$$
a^{\prime}=\sum_{i=1}^{k-1} \sigma_{(i: k)}^{2} \quad \text { and } \quad b^{\prime}=\sigma_{(k: k)}^{2} .
$$

The relative precision of $\bar{Y}_{(k) t}$ relative to SRS is 


\section{BHOJ \& CHANDRA}

$$
R P_{\mathrm{SRS}: \mathrm{t}}=\frac{k^{2} \sigma^{2}}{(k-1+t)\left(a^{\prime}+b^{\prime} / t\right)},
$$

and the relative precision of $\bar{Y}_{(k) t}$ with respect to RSS with equal allocation is

$$
R P_{\text {eql:t }}=\frac{k^{2} \overline{\sigma^{2}}}{(k-1+t)\left(a^{\prime}+b^{\prime} / t\right)} .
$$

Optimizing (13), the optimal value of $t$ is

$$
t_{\mathrm{opt}}=\sqrt{\frac{b^{\prime}(k-1)}{a^{\prime}}} .
$$

In $(s, t)$-model, two largest order statistics are assigned more weight than others. In $(s, t)$ model,

$$
m^{\prime \prime} \equiv m_{1}=m_{2}=\ldots=\frac{m_{k-1}}{s}=\frac{m_{k}}{t}, \quad 1 \leq s \leq t,
$$

with the sample size $n=(k-2+s+t) m^{\prime \prime}$.

The variance of the estimator under $(s, t)$-model is

$$
\operatorname{Var}\left(\bar{Y}_{(k) s t}\right)=\frac{1}{k^{2} m^{\prime \prime}}(a+b / s+c / t)
$$

where

$$
a=\sum_{i=1}^{k-2} \sigma_{(i: k)}^{2}, \quad b=\sigma_{(k-1: k)}^{2}, \quad \text { and } \quad c=\sigma_{(k: k)}^{2}
$$

The relative precisions of the $(s, t)$-model with respect to SRS and RSS with equal allocation are

$$
R P_{\mathrm{SRS}: \mathrm{st}}=\frac{k^{2} \sigma^{2}}{(k-2+s+t)(a+b / s+c / t)},
$$


UNEQUAL ALLOCATION FOR RSS FOR SKEW DISTRIBUTIONS

$$
R P_{\text {eql:st }}=\frac{k^{2} \overline{\sigma^{2}}}{(k-2+s+t)(a+b / s+c / t)} .
$$

The optimum value of $(s, t)$ after optimizing (18) with respect to $s$ and $t$ is given by

$$
s^{*}=\sqrt{\frac{(k-2) b}{a}} \text { and } t^{*}=\sqrt{\frac{(k-2) c}{a}} \text {. }
$$

The $t$-model, $(s, t)$-model, and Neyman's model do not generally give integer values of $m_{i}$. Therefore, some adjustments are necessary to obtain the appropriate optimal solution. The values of $m_{i}$ are approximated here to the nearest integer values. This rule is not observed if $m_{i}<1$. In some cases, further adjustments may be required so that the same sample size can be obtained for all unequal allocation models. For this reason, the following rule is used. The adjustments in the $t$-model are made only in $m_{k}$ because of equal $m_{i}=1,2, \ldots,(k-1)$. In the case of $(s, t)$-model, the first $(k-2)$ values of samples are equal. Therefore, the adjustments in $m_{k-1}$ and $m_{k}$ are based on the following rule. If the adjustment in the total sample size is of one unit increase (decrease), the adjustment is made in order statistic with highest (lowest) fractional value. If the adjustment in the sample size $n$ is to be made of two units increase (decrease), the adjustments are made in both unequal samples of order statistics. In the unusual case where the adjustment of more than two units is needed, the above rules can be modified.

Consider another optimal unequal allocation model on the assumption that the population standard deviations of order statistics are known:

$$
m_{i}=\frac{\sigma_{(i: k)}}{\sigma_{(1: k)}}
$$

with sample size

$$
n=\sum_{i=1}^{k} m_{i} .
$$

Then the variance of the estimator of $\mu$ can be obtained from equation (5). It can be easily shown by using 


\section{BHOJ \& CHANDRA}

$$
\sigma_{(1: k)}=\frac{\sum_{i=1}^{k} \sigma_{(i: k)}}{n}
$$

that the variance of this estimator is the same as for Neyman's optimal solution. The advantage of this approach is that it sets the value of $m_{1}=1$. However, it also fixes the value of sample size. The disadvantages of this approach are the same as those of Neyman's allocation model. In the next section we introduce simple unequal allocation model which is not based on unrealistic assumptions about the distributions.

\section{Simple Unequal Allocation Models}

Tiwari and Chandra (2011) proposed a systematic approach for unequal allocation models where $m_{i}=i, i=1,2, \ldots, k$ with $n=k(k+1) / 2$. They showed the relative precision of their estimator is better than those of $t$-model for moderately positive skewed distributions. Furthermore, their relative precisions are quite close to $(s, t)$-model. However, this model does not work well for positively skewed distributions with heavy right tails.

The aim of this study is to propose a simple and accurate model for unbalanced RSS for skew distributions with heavy right tails. The proposed unequal allocation model is to use the unbiased estimator for the population mean given by (4) with

$$
\begin{gathered}
m_{i}=i, i=1,2, \ldots,(k-1), m_{k}=\left(k^{2}+1-\frac{k(k-1)}{2}\right), \quad k \leq 4, \text { and } \\
m_{i}=i, i=1,2, \ldots,(k-2), m_{k-1}=k, m_{k}=\left(k^{2}-\frac{k(k-1)}{2}\right), \quad k>4 .
\end{gathered}
$$

The resulting sample size of this model is $n=k^{2}+1$. 


\section{UNEQUAL ALLOCATION FOR RSS FOR SKEW DISTRIBUTIONS}

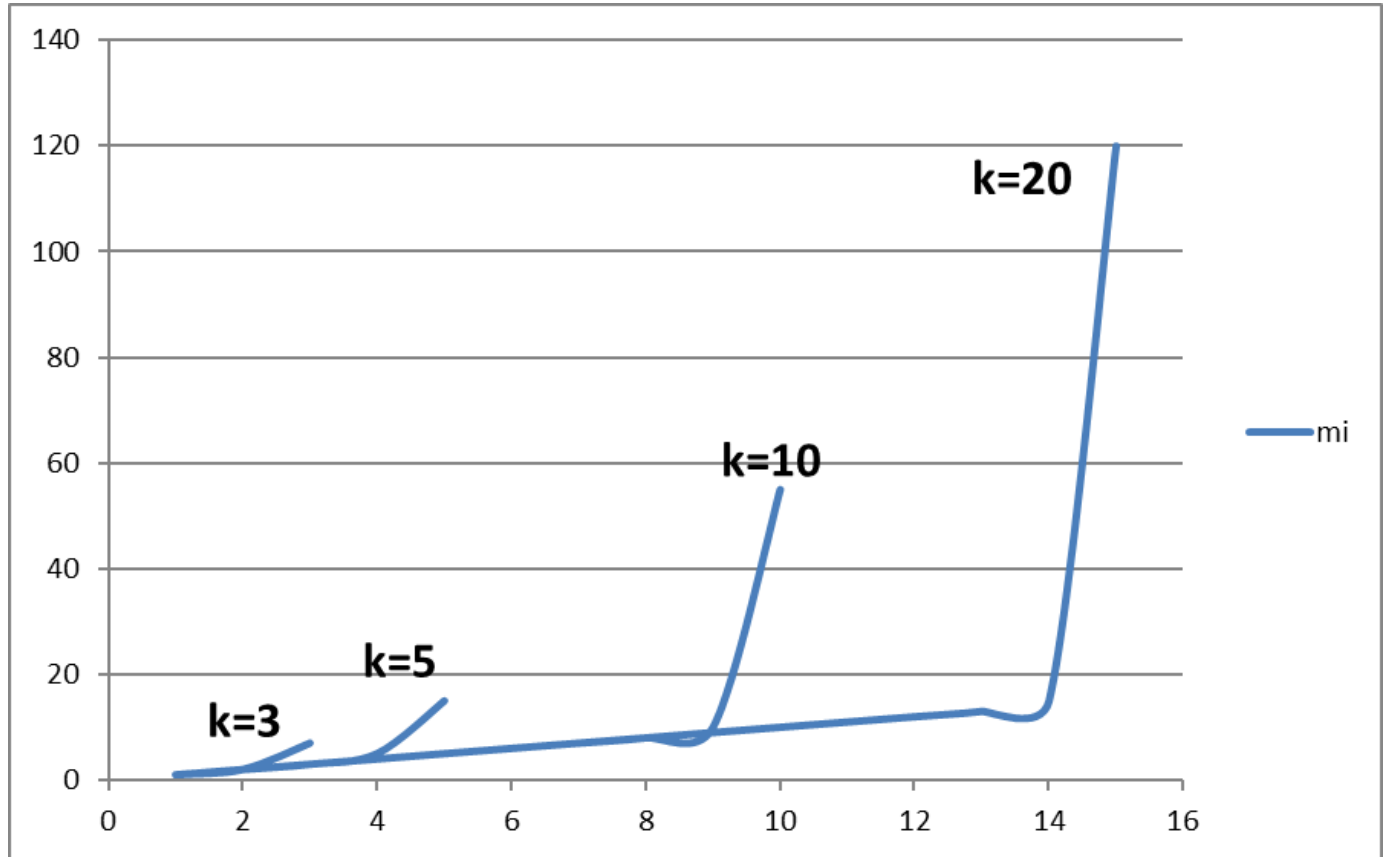

Figure 1. Relation between the order statistic and corresponding allocations at $k=3,5$, 10 , and 20

The relative precision of this simple unequal allocation model with respect to $\mathrm{SRS}$ is

$$
R P_{\text {SRS:simp }}= \begin{cases}\frac{k^{2} \sigma^{2}}{\left(k^{2}+1\right)\left(\sum_{i=1}^{k-1} \frac{\sigma_{(i: k)}^{2}}{i}+\frac{2 \sigma_{(k: k)}^{2}}{\left(k^{2}+k+2\right)}\right)}, & k \leq 4 \\ \frac{k^{2} \sigma^{2}}{\left(k^{2}+1\right)\left(\sum_{i=1}^{k-1} \frac{\sigma_{(i: k)}^{2}}{i}+\frac{\sigma_{(k-1: k)}^{2}}{k}+\frac{2 \sigma_{(k: k)}^{2}}{\left(k^{2}+k\right)}\right)}, & k>4\end{cases}
$$

The model does not use any information about the parameters or standard deviations of the order statistics of the skew distributions. This method seems to be useful in forestry, environmental, agricultural, medical and other allied areas, as the number of replications corresponding to each order statistic for measurement could be easily decided. 


\section{BHOJ \& CHANDRA}

\section{Comparison of Estimators}

The performance of our estimator for population mean based on unequal allocation model will be compared with estimators based on balanced RSS, $t$-model, $(s, t)$ model, Neyman's optimal unequal allocation model for the five positively skewed distributions with heavy right tail. These distributions are Lognormal $(\operatorname{LN}(0,1))$, Pareto $(\mathrm{P}(3), \mathrm{P}(4), \mathrm{P}(5))$ and Weibull $(\mathrm{W}(0.5))$. For these distributions, the means and variances of order statistics are readily available in Harter and Balakrishnan (1996). The relative precisions for these distributions were computed for various set sizes by using the balanced RSS, $t$-model, $(s, t)$-model, Neyman's optimal allocation model and our simple unequal allocation model, and they are presented in Table 1. The proposed model is superior to the balanced RSS model.

Table 1. Comparison of relative precisions

\begin{tabular}{rrrrrrrrr} 
& & \multicolumn{7}{c}{ Set size $(\boldsymbol{k})$} \\
\cline { 3 - 8 } Distribution & Model & $\mathbf{2}$ & $\mathbf{3}$ & $\mathbf{4}$ & $\mathbf{5}$ & $\mathbf{6}$ & $\mathbf{7}$ & $\mathbf{8}$ \\
\hline LN(0,1) & $R P_{\text {SRS:eql }}$ & 1.1872 & 1.3393 & 1.4711 & 1.5891 & 1.6971 & 1.7974 & 1.8914 \\
& $R P_{\text {SRS:t }}$ & 1.5765 & 1.9935 & 2.4107 & 2.7834 & 3.0756 & 3.3653 & 3.6647 \\
& $R P_{\text {SRS:st }}$ & & 2.1182 & 2.5242 & 3.0491 & 3.4218 & 3.8388 & 4.2002 \\
& $R P_{\text {SRS:opt }}$ & 1.5765 & 2.1182 & 2.6219 & 3.1347 & 3.6193 & 4.0898 & 4.5588 \\
& $R P_{\text {SRS:simp }}$ & 1.5765 & 2.1182 & 2.6219 & 3.1347 & 3.5975 & 4.0189 & 4.4058 \\
$\mathrm{P}(3)$ & $R P_{\text {SRS:eql }}$ & 1.1364 & 1.2422 & 1.3305 & 1.4072 & 1.4755 & 1.5373 & 1.5941 \\
& $R P_{\text {SRS:t }}$ & 1.6000 & 2.0039 & 2.4896 & 2.8017 & 3.1699 & 3.4769 & 3.7247 \\
& $R P_{\text {SRS:st }}$ & & 2.1505 & 2.6441 & 3.0894 & 3.5209 & 3.9029 & 4.2872 \\
& $R P_{\text {SRS:opt }}$ & 1.6000 & 2.1505 & 2.6441 & 3.1671 & 3.6790 & 4.1762 & 4.6584 \\
& $R P_{\text {SRS:simp }}$ & 1.6000 & 2.1505 & 2.6713 & 3.1511 & 3.6530 & 4.1182 & 4.5529 \\
$\mathrm{P}(4)$ & $R P_{\text {SRS:eql }}$ & 1.1951 & 1.3547 & 1.4927 & 1.6162 & 1.7287 & 1.8329 & 1.9305 \\
& $R P_{\text {SRS:t }}$ & 1.5681 & 1.9897 & 2.3798 & 2.7628 & 3.0648 & 3.3359 & 3.6479 \\
& $R P_{\text {SRS:st }}$ & & 2.1037 & 2.5123 & 3.0130 & 3.3953 & 3.7774 & 4.1509 \\
& $R P_{\text {SRS:opt }}$ & 1.5681 & 2.1037 & 2.6111 & 3.1171 & 3.5994 & 4.0703 & 4.6660 \\
& $R P_{\text {SRS:simp }}$ & 1.5681 & 2.1037 & 2.6002 & 3.1171 & 3.5729 & 3.9912 & 4.3802 \\
$\mathrm{P}(5)$ & $R P_{\text {SRS:eql }}$ & 1.2277 & 1.4179 & 1.5861 & 1.7390 & 1.8797 & 2.0126 & 2.1373 \\
& $R P_{\text {SRS:t }}$ & 1.5439 & 1.9919 & 2.3179 & 2.7242 & 3.0572 & 3.3497 & 3.6072 \\
& $R P_{\text {SRS:st }}$ & & 2.0601 & 2.5098 & 2.9560 & 3.3727 & 3.7209 & 4.0975 \\
& $R P_{\text {SRS:opt }}$ & 1.5439 & 2.0601 & 2.5847 & 3.0724 & 3.5473 & 4.0340 & 4.4923 \\
& $R P_{\text {SRS:simp }}$ & 1.5439 & 2.0601 & 2.5374 & 3.0611 & 3.4841 & 3.8857 & 4.2446 \\
& $R P_{\text {SRS:eql }}$ & 1.1268 & 1.2362 & 1.3345 & 1.4250 & 1.5094 & 1.5891 & 1.6648 \\
& $R P_{\text {SRS:t }}$ & 1.6306 & 2.0625 & 2.5008 & 2.7847 & 3.0754 & 3.3506 & 3.5747 \\
& $R P P_{\text {SRS:st }}$ & & 2.2105 & 2.7913 & 3.1900 & 3.6799 & 3.9897 & 4.4030 \\
& $R P_{\text {SRS:opt }}$ & 1.6306 & 2.2105 & 2.7913 & 3.3840 & 3.9393 & 4.4796 & 5.0320 \\
& $R P_{\text {SRS:simp }}$ & 1.6306 & 2.2105 & 2.7522 & 3.2696 & 3.7754 & 4.2242 & 4.6243 \\
\hline $\mathrm{W}(0.5)$
\end{tabular}




\section{UNEQUAL ALLOCATION FOR RSS FOR SKEW DISTRIBUTIONS}

The relative precisions of $t$-model, Neyman's model, and our proposed model are the same for $k=2$. The proposed simple model is better than $t$-model for all $k>2$. In addition, it is better than or equal to $(s, t)$-model for $k=3$ and 4 with one exception. However, the simple model is uniformly better than $(s, t)$-model for $k>4$. The relative precision of all estimators increases as the set size $k$ and hence sample size $n$ increases.

Consider the performance of the above all unequal allocation models with the increasing values of skewness of a family of distributions. Consider the skew distribution $\mathrm{LN}(1, \sigma) ; X \sim \mathrm{LN}(\mu, \sigma)$ with pdf

$$
\mathrm{f}(x)=\frac{1}{x \sigma \sqrt{2 \pi}} \exp \left[\frac{-1}{2}\left(\frac{\log x-\mu}{\sigma}\right)^{2}\right], \quad x>0, \mu>0, \sigma>0 \text {. }
$$

Then skewness $(S k)$ and shape parameter $(p)$ are given by

$$
\begin{aligned}
S k & =\sqrt{\beta_{1}}=\sqrt{\exp \left(\sigma^{2}\right)-1}\left(\exp \left(\sigma^{2}\right)+2\right) \\
p & =\exp \left(\sigma^{2}\right)
\end{aligned}
$$

The performance of unequal allocation models relative to SRS with $k=5$ is presented in Table 2 for lognormal family of distributions for a range of values of

Table 2. Relative precisions of unequal allocation models for $\operatorname{Lognormal} \mathrm{LN}(1, \sigma)$ distributions for $k=5$

\begin{tabular}{rrrrrrrr}
$\begin{array}{r}\text { Shape } \\
\text { parameter }\end{array}$ & $\boldsymbol{\sigma}$ & $\boldsymbol{S k}\left(\sqrt{\boldsymbol{\beta}_{1}}\right)$ & $\boldsymbol{R} \boldsymbol{P}_{\text {SRS:eql }}$ & $\boldsymbol{R} \boldsymbol{P}_{\text {SRS: } t}$ & $\boldsymbol{R} \boldsymbol{P}_{\text {SRS:st }}$ & $\boldsymbol{R} \boldsymbol{P}_{\text {SRS:simp }}$ & $\boldsymbol{R} \boldsymbol{P}_{\text {SRS:opt }}$ \\
\hline 1.8 & 0.77 & 3.40 & 1.8888 & 2.6862 & 2.9037 & 2.8616 & 2.9626 \\
1.9 & 0.80 & 3.70 & 1.8402 & 2.7043 & 2.9054 & 2.9159 & 2.9858 \\
2.0 & 0.83 & 4.00 & 1.7971 & 2.7197 & 2.9085 & 2.9612 & 3.0031 \\
2.1 & 0.86 & 4.30 & 1.7586 & 2.7328 & 2.9352 & 2.9993 & 3.0216 \\
2.2 & 0.89 & 4.60 & 1.7241 & 2.7441 & 2.9575 & 3.0314 & 3.0373 \\
2.3 & 0.91 & 4.90 & 1.6928 & 2.7539 & 2.9762 & 3.0586 & 3.0586 \\
2.4 & 0.94 & 5.21 & 1.6645 & 2.7625 & 2.9985 & 3.0816 & 3.0816 \\
2.5 & 0.96 & 5.51 & 1.6386 & 2.7700 & 3.0168 & 3.1012 & 3.1012 \\
2.6 & 0.98 & 5.82 & 1.6148 & 2.7766 & 3.0328 & 3.1180 & 3.1180 \\
2.7 & 1.00 & 6.13 & 1.5929 & 2.7824 & 3.0468 & 3.1323 & 3.1323 \\
2.8 & 1.01 & 6.44 & 1.5727 & 2.7875 & 3.0590 & 3.1445 & 3.1445 \\
2.9 & 1.03 & 6.75 & 1.5540 & 2.7920 & 3.0699 & 3.1549 & 3.1549 \\
3.0 & 1.05 & 7.07 & 1.5366 & 2.7961 & 3.0794 & 3.1639 & 3.1639 \\
\hline
\end{tabular}




\section{BHOJ \& CHANDRA}

population standard deviation. The variances of the order statistics of the family of distributions were computed by using the variances of order statistics for different values of shape parameter which are readily available in Balakrishnan and Chen (1999). From Table 2, it can be seen that as skewness increases the performance of equal allocation model decreases. The relative precisions of our estimator are better than those based on $t$-model for all values of $\sigma$. The relative precisions of our model are higher than those based on $(s, t)$-model when $\sigma>0.80$. Most importantly, for $\sigma \geq 0.91$, the relative precisions based on our simple model are the same as those based on Neyman's allocation model.

\section{Conclusion and Discussion}

A simple and precise unequal allocation model for ranked set sampling was proposed when distributions under consideration are positively skew with very heavy right tails. It does not use any unrealistic assumptions such as the measures of skewness, measures of kurtosis or the standard deviations of order statistics. The estimator of the population mean based on our simple method is compared with the estimators based on equal allocation model, $t$-model, $(s, t)$-model and Neyman's optimal method. The last three methods are harder to use, because they all are based on the unrealistic assumption that the population standard deviations of order statistics are known. However, even in this unfavorable and disadvantageous situation the proposed simple model performs better than the other unequal allocation models.

The relative precisions for $k=2$ are equal for $t$-model, the proposed model, and Neyman's allocation model for all five distributions. However, the relative precisions of our estimator are higher than those based on $t$-model for $k>2$. In addition, the relative precisions of our model are the same as those of $(s, t)$-model for $k=3$. The relative precisions of our estimator are higher than those based on $(s, t)$-model for $k=4$ except for $\mathrm{W}(0.5)$ distribution. In this case, the relative precision of $(s, t)$-model is the same as the one based on Neyman's optimal allocation model. Furthermore, the proposed simple model is uniformly better than $(s, t)$-model for $k>4$. There are some cases where its relative precisions are the same as those based on Neyman's approach, particularly for $k=2$ and 3 for all five selected distributions. It also happens for $k=4$ and $k=5$ for $\operatorname{LN}(0,1)$ and for $k=5$ for $\mathrm{P}(4)$ as can be seen from Table 1 . This is also true for the family of lognormal distributions when $\sigma \geq 0.91$ in Table 2. In two instances in two tables our relative precision is slightly greater than the one based on Neyman's allocation. This might happen because Neyman's approach may lose its optimal property when the sample 


\section{UNEQUAL ALLOCATION FOR RSS FOR SKEW DISTRIBUTIONS}

sizes are approximated by complete integers. Based on the numerical computations of relative precisions, the proposed simple unequal allocation model is recommended for estimation of population mean of skew distributions with heavy right tails for all values of $k$.

\section{References}

Balakrishnan, N., \& Chen, W. S. (1999). Handbook of tables of order statistics from lognormal distribution with applications. New York: Springer. doi: 10.1007/978-1-4615-5309-0

Bhoj, D. S. (1997). New parametric ranked set sampling. Journal of Applied Statistical Sciences, 6(4), 275-289.

Bhoj, D. S. (2001). Ranked set sampling with unequal samples. Biometrics, 57(3), 957-962. doi: 10.1111/j.0006-341X.2001.00957.x

Bhoj, D. S., \& Ahsanullah, M. (1996). Estimation of the parameters of the generalized geometric distribution using ranked set sampling. Biometrics, 52(2), 685-694. doi: 10.2307/2532906

Bhoj, D. S., \& Kushary, D. (2016). Ranked set sampling with unequal samples for skew distributions. Journal of Statistical Computations and Simulations, 86(4), 676-681. doi: 10.1080/00949655.2015.1028405

Chen, Z., Bai, Z. D., \& Sinha, B. K. (2004). Ranked set sampling: Theory and applications. New York: Springer-Verlag. doi: 10.1007/978-0-387-21664-5

Cobby, J. M., Ridout, M. S., Bassett, P. J., \& Large, R.V. (1985). An investigation into the use of ranked set sampling on grass and grass-clover swards. Grass and Forage Science, 40(3), 257-263. doi: 10.1111/j.13652494.1985.tb01753.x

Dell, T. R., \& Clutter, J. L. (1972). Ranked set sampling theory with order statistics background. Biometrics, 28(2), 545-555. doi: 10.2307/2556166

Halls, L. K., \& Dell, T. R. (1966). Trials of ranked set sampling for forage yields. Forest Science, 12(2), 22-26. doi: 10.1093/forestscience/12.1.22

Harter, H. L., \& Balakrishnan, N. (1996). CRC handbook of tables for the use of order statistics in estimation. Boca Raton, FL: CRC Press.

Johnson, G. Q., Paul, G. P., \& Sinha, A. K. (1993). Ranked set sampling for vegetation research. Abstracta Botanica, 17, 87-102. 


\section{BHOJ \& CHANDRA}

Kaur, A., Patil, G. P., \& Taillie, C. (1997). Unequal allocation models for ranked set sampling with skew distributions. Biometrics, 53(1), 123-130. doi: $10.2307 / 2533102$

Lam, K., Sinha, B. K., \& Zhong, W. (1994). Estimation of parameters of the two-parameter exponential distribution using ranked set sample. Annals of the Institute Statistical Mathematics, 46, 723-736. doi: 10.1007/BF00773478

McIntyre, G. A. (1952). A method for unbiased selective sampling using ranked sets. Australian Journal of Agricultural Research, 3(4), 385-390. doi: 10.1071/AR9520385

Neyman, J. (1934). On the two different aspects of the representative method: The method of stratified sampling and the method of purposive selection. Journal of the Royal Statistical Society, 97(4), 558-625. doi: 10.1111/j.23972335.1934.tb04184.x

Patil, G. P., Sinha, A. K., \& Taillie, C. (1994). Ranked set sampling. In G. P. Patil \& C. R. Rao (Eds.), Handbook of statistics 12: Environmental statistics (pp. 167-198). Amsterdam: Elsevier. doi: 10.1016/S0169-7161(05)80007-0

Stokes, S. L. (1977). Ranked set sampling with concomitant variables. Communications in Statistics - Theory and Methods, 6(12), 1207-1211. doi: 10.1080/03610927708827563

Stokes, S. L., \& Sager, T. W. (1988). Characterization of a ranked set sample with application to estimating distribution functions. Journal of the American Statistical Association, 83(402), 374-381. doi:

10.1080/01621459.1988.10478607

Takahasi, K., \& Wakimoto, K. (1968). On unbiased estimates of the population mean based on the sample stratified by means of ordering. Annals of the Institute of Statistical Mathematics, 20(1), 1-31. doi: 10.1007/BF02911622

Tiwari, N., \& Chandra, G. (2011). A systematic procedure for unequal allocation for skewed distributions in ranked set sampling. Journal of the Indian Society of Agricultural Statistics, 65(3), 331-338. 